\title{
Singularity Intensity Factor Calculation in Plates and Shells Based on the Singular Line Mapping Technique in FEMOL
}

\author{
Chuntu Liu and Yongjun $\mathrm{Xu}$ \\ Institute of Mechanics, Chinese Academy of Sciences, \\ Beijing 100080, China P.R.
}

Keywords: Finite Element Method of Lines, Reissner Plate, Reissner Shell, Singular Line Mapping, Stress Intensity Factor

\begin{abstract}
In this paper, a brief review of the singular line mapping technique in finite element method of lines (FEMOL) for plate and shell crack/notch problems is given. By using the singular line mapping technique, the solution property is changed, the computation is speeded up and the accuracy is improved. The displacement and its derivative can be obtained accurately after an accurate singular line mapping, and the lowest stress intensity factor can be solved from the derivative. Some numerical examples are given to show the general excellent performance.
\end{abstract}

\section{INTRODUCTION}

Stress analysis in practical engineering applications inevitably encounters stress singularities caused by sudden changes in geometry, e.g. around re-entrant corners (notches) or, more severely, around crack tips. Their presence causes great difficulty to the numerical solutions that have to be invoked when analytical solutions are not available. A numerical recipe for accurate and efficient computation of stress singularity factors (SIFs) usually consists of two major ingredients, namely a powerful numerical method for general stress analysis and a novel approach to obtaining the desired SIFs which may include special treatment of various singularities. The underlying numerical method employed in this paper is the FEMOL [1-4], which is a newly developed, general-purpose, semi-analytical method. In this method, a partial differential equation defined on an arbitrary domain is semi-discretized, by finite element techniques via energy theorems or variational principles, into a system of ordinary differential equations (ODEs) defined on straight or curved mesh lines.At present, the resulting ODE system is solved directly and efficiently by state-of-the-art ODE solver, e.g. COLSYS $[5,6]$ is exclusively adopted in the present paper. Such solvers have built-in self-adaptivity features such that the accuracy of the ODE solutions satisfies the user pre-specified error tolerances. With the solver used, FEMOL has been proved to be a distinguished numerical method with efficient adaptivity in the mesh line directions automatically built in, so that its inherent semi-analytical characteristics are well preserved. Its power and versatility have been demonstrated by a series of theoretical analysis and computational applications to various linear and nonlinear problems. A general-purposed computer code FEMOL92 [7] that is capable of static and vibrative analysis of various linear elastic structures has been developed. For a more detailed and systematic description of FEMOL, see Ref. [4]. FEMOL could be directly applied to notch/crack 
problems [3,4]. The success in establishing 'triangular' elements (Yuan [8]) by degenerating an element end-side to a point allows the use of the optimum radial line meshes, i.e. mesh lines emanate from crack tips. With no special treatment, except for judicious use of radial line meshes with sufficiently high polynomial degree $p$ used in the element displacement shape functions, FEMOL is able to produce remarkably accurate solutions even when very few elements are used [4] Most of numerical methods, directly or indirectly, make use of the available eigen-solutions when applied to the SIF computation for crack/notch problems so that singularity can be treated in a more efficient, accurate and reliable way. Recently, $\mathrm{Xu}$ and Yuan [9] presented an effective implementation of the imbedding method for accurate and reliable computation of complete real or complex eigen-solutions in two dimensional notch/crack singularities with multiple materials, arbitrary opening angles and various surface conditions. The resulting algorithm is robust and may be employed by any numerical methods that make use of singular solutions.

In order to take full advantage of the power of FEMOL and the usefulness of the local singular solutions, the singular mapping technique [10] is utilized in this paper to remove the singularity from the FEMOL solving to the mapping. This nonlinear mapping not only can map an interval to a standard one, but also can change the behavior of the solution function. Combined with the selfadaptivity features, the computation is speeded up and the accuracy is improved, especially, the tolerances can be imposed on the derivatives as the displacement for the singular solutions. The displacement and its derivative can be obtained accurately after an accurate singular line mapping, and from the derivative we can solve the lowest stress intensity factor. Recently, Xu and Yuan [11] use this technique in anti-plane and in-plane problems to solve the SIFs. In the present paper, a brief review of the singular line mapping technique in finite element method of lines (FEMOL) for plate and shell crack/notch problems is given. By using the singular line mapping technique, both the displacement and its derivative can be obtained accurately after an accurate singular line mapping. and from derivative the lowest stress intensity factor can be solved. Some numerical examples are given to show the general excellent performance.

\section{SINGULAR MAPPING TECHNIQUE OF FEMOL}

Figure 1 shows a two dimensional singular problem with $N$ different materials around notch/crack tip. The body forces are assumed to be negligible. For convenience sake we omit the different material domain subscript $j$ shown in the Fig. 1(a). In the polar coordinates shown in Fig. 1(a), for the Reissner plate and shell, the general displacement components can be written as the following double series form

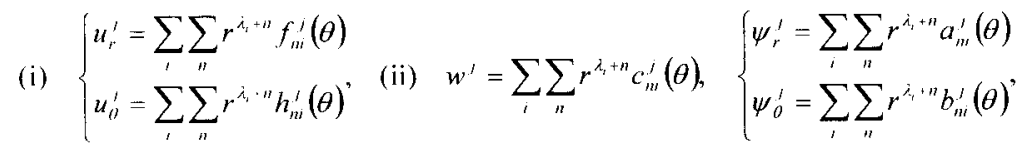

part (i) is omitted for the Reisser plate. It shows that the singularity may appear when $n=0$ and in the first two or three terms of $i$. Here we only take into account of the main part of $n=0$, the Reissner plate and Reissner shell could be divided into two basic parts of anti-plane part and inplane part. The well-known Williams' displacement $w_{0}(r, \theta)$ and potential function $\phi_{0}(r, \theta)$ are

$$
\text { (i) } w_{0}(r, \theta)=\sum_{, 0,1,2,3, \ldots} \alpha_{i} r^{\lambda_{1}} f_{i}(\theta) . \quad \text { (ii) } \phi_{0}(r, \theta)=\sum_{, 0,1,2, \ldots} \alpha_{i} i^{\lambda_{1}, 1} F,(\theta)
$$

where both $f_{1}(\theta)$ and $F_{,}(\theta)$ are certain normalization eigen-functions corresponding to the eigenvalue $\lambda_{1}$, and the well known explicit forms are 


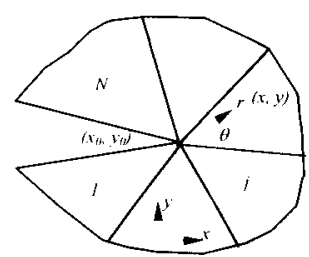

(a) Original problem

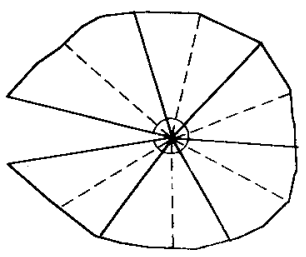

(b) FEMOL mesh with radial lines

Fig. $1 \mathrm{~N}$-material notch problem

$f_{i}(\theta)=A_{i} \cos \lambda_{1} \theta+B_{i} \sin \lambda_{i} \theta, \quad F_{i}(\theta)=A_{i} \sin \left(\lambda_{1}+1\right) \theta+B_{i} \cos \left(\lambda_{i}+1\right) \theta+C_{i} \sin \left(\lambda_{1}-1\right) \theta+D_{i} \cos \left(\lambda_{1}-1\right) \theta$

where $\left\{A_{i}, B_{i}\right\}$ and $\lambda_{i}$ in $f_{i}(\theta)$ are real constants, $\left\{A_{i}, B_{i}, C_{i}, D_{i}\right\}$ and $\lambda_{i}$ in $F_{i}(\theta)$ are complex constants. $\left(u_{r i_{i}}, u_{00_{1}}\right)$ and $\left(\psi_{r 0 i}, \psi_{00_{l}}\right)$ can be derived from the stress potential function

$$
u_{r(0,}, \psi_{r(t)}=r^{\lambda} \frac{\left(1-v \lambda_{1}\right)\left(1+\lambda_{i}\right) F_{i}(\theta)+F_{i}(\theta)}{\lambda E} u_{\theta 0,}, \psi_{\theta 0 t}=r^{\lambda} \frac{\left(1+\lambda_{i}-\left(1-\lambda_{1}\right) \lambda_{i} v+2 \lambda_{1}^{2}\right) F_{i}^{\prime}(\theta)+F_{i}^{\prime}(\theta)}{\lambda(1-\lambda) E}
$$

the corresponding stress components of both anti-plane and plane problem are

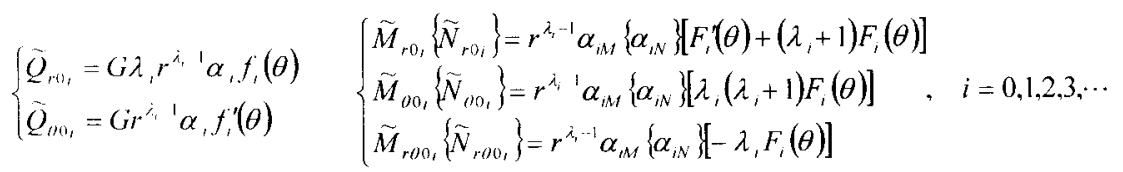

when $r \rightarrow 0$, the stresses will tend to infinity because there are singular terms $r^{i_{1} \cdot 1}$, the same singularity will exist in the derivative when implement FEMOL. When the following variable substitution is taken, the singularity that in the derivative and the strain or stresses will be removed

$$
\rho=r^{\lambda_{1}}
$$

where $\lambda_{1}$ is the first eigne-value. The corresponding expansions can be rewritten as

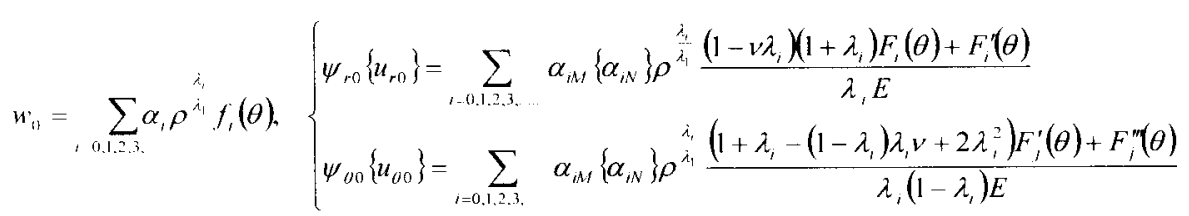

solving derivative of variable $r$ is replaced by solving the derivative term of variable $\rho$, and the singularity is removed. To achieve this ideal situation in FEMOL, we judiciously choose a radial line mesh as shown in Fig. $1 \mathrm{~b}$. For the $n$-th line, the mapping and the inverse mapping relations are

$$
\eta=-1+2\left(\frac{r}{L_{n}}\right)^{\lambda_{1}}, \quad r=L_{n}\left(\frac{\eta+1}{2}\right)^{\lambda_{1}}
$$

After singular line mapping, the Williams expansions in formula (7) will be changed as

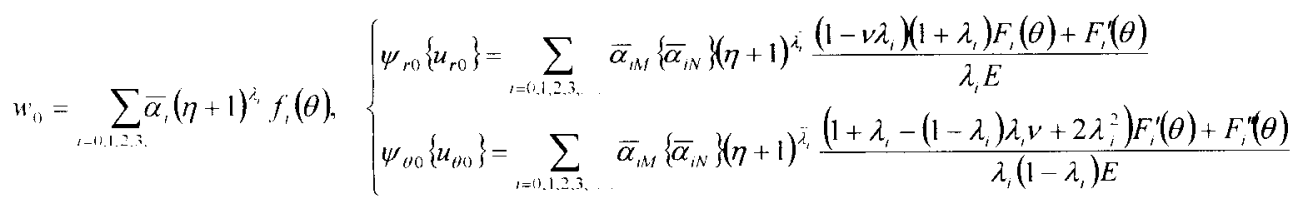

where $\bar{\lambda}_{1}=\lambda_{1} / \lambda_{1}, \bar{\alpha}_{1}=\alpha_{1} L_{n}^{i_{n}} / 2^{\lambda_{1}}$ 


\section{COMPUTATION FOR THE LOWEST SIF}

For both the anti-plane and the in-plane parts, to seek the SIFs is tantamount to find the corresponding coefficients $\alpha$, of the Williams expansions in (2), especially the lowest coefficient $\alpha_{1}$. From the formula (9) we know that, if the coefficients of $\bar{\alpha}_{i}$ are known, the coefficients $\alpha$, are obtained too

$$
\alpha_{0}=\bar{\alpha}_{0}, \quad \alpha_{1}=2 \bar{\alpha}_{1} / L_{n}^{\lambda_{1}}
$$

From FEMOL solutions, the displacement terms and the derivative can be easily calculated, then we can get the lowest one or two coefficients by using the following way

(i) $\bar{\alpha}_{N N}=\lim _{\eta \rightarrow 1} \frac{\partial^{\prime} u_{r 0}(\eta, \theta)}{\partial \eta^{\prime}} / \delta_{1}$, or $\quad \bar{\alpha}_{j N}=\lim _{\eta \rightarrow-1} \frac{\partial^{\prime} u_{v 0}(\eta, \theta)}{\partial \eta^{\prime}} / \delta_{2,}, \quad j=0,1$

(ii) $\left\{\begin{array}{l}\bar{\alpha}_{0}=\lim _{\eta \rightarrow 1} w_{i 0}(\eta, \theta) / f_{0}(\theta), \quad \bar{\alpha}_{1}=\lim _{\eta \rightarrow-1} \frac{\partial w_{0}(\eta, \theta)}{\partial \eta} / f_{1}(\theta) \\ \bar{\alpha}_{\mu^{\prime \prime}}=\lim _{\eta \rightarrow 1} \frac{\partial^{\prime} \psi_{r 0}(\eta, \theta)}{\partial \eta^{\prime}} / \delta_{1}, \text { or } \quad \bar{\alpha}_{i M}=\lim _{\eta \rightarrow-1} \frac{\partial^{\prime} \psi_{\nu 0}(\eta, \theta)}{\partial \eta^{\prime}} / \delta_{2,}, \quad j=0,1\end{array}\right.$

where, without losing the generality, we use $\mathrm{d}^{0} / \mathrm{d}()^{\circ}$ to indicate the displacement and

$$
\begin{aligned}
& \delta_{10}=\frac{4 D_{0} \cos (\theta)-(1-v) F_{0}(\theta)}{E}, \quad \delta_{20}=\frac{-4 D_{0} \sin (\theta)-(1-v) F_{0}^{\prime}(\theta)}{E} \\
& \delta_{11}=\frac{\left(1-v \lambda_{1}\right)\left(1+\lambda_{1}\right) F_{1}(\theta)+F_{1}(\theta)}{\lambda_{1} E}, \quad \delta_{21}=\frac{\left(1+\lambda_{1}-\left(1-\lambda_{1}\right) \lambda_{1} v+2 \lambda_{1}^{2}\right) F_{1}^{\prime}(\theta)+F_{1}^{\prime \prime}(\theta)}{\lambda_{1}\left(1-\lambda_{1}\right) E}
\end{aligned}
$$

It is well known that the coefficients of the $r^{\lambda_{1}-1}$-stress singularity are related to the stress intensity factors, From the relationship between the coefficients and the stresses, the SIFs are generally defined as

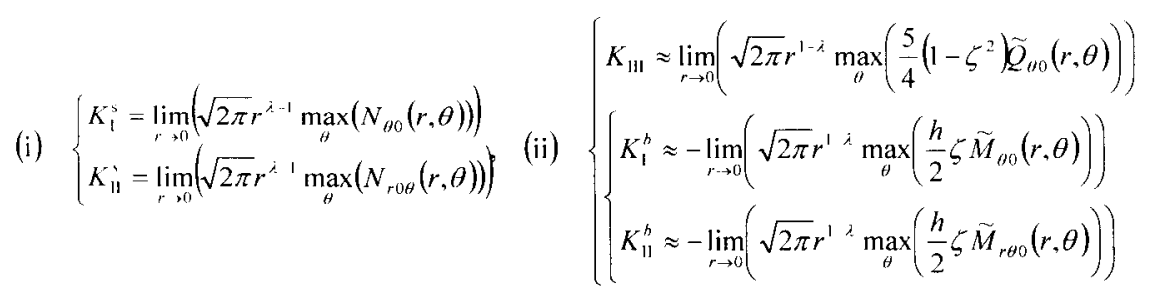

where superscript $s$ indicates the membrane stress part, $b$ indicates bending part.

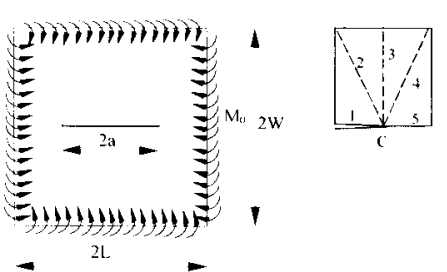

(a) Original problem (b) A quarter domain and the FEMOL meshes

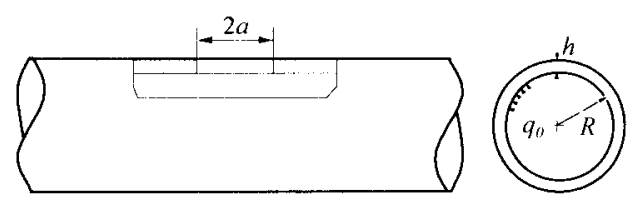

Fig. 3 A pressurized cylindrical shell with a longitudinal crack

Fig. 2 Reissner plate with a center 


\section{NUMERICAL EXAMPLES}

To assess the performance of the proposed method, some numerical examples are given in this section. The following notations are used

$L_{t}-i$-th line on which the SIFs are calculated

$L_{n}$ - the line length

$p$ - polynomial degree used for element displacements

$T O L-$ tolerance specified for ODE solutions.

In the following examples, all FEMOL tolerances are taken as $T O L=0.01 \%$.

Example 1 Reissner plate with a center crack

In this example, as Fig. 2(a) shows, we consider a Reissner plate problem with a center crack. A quarter body was taken in computing by using the symmetry as Fig. 2(b) shows. The necessary constants are $M_{0}=1.0, E=1.0^{7}$ and $v=0.3$, the length of crack is $2 a$ and the thickness of the plate is $h, 2 L=2 W=4 a$, with $a=1$. Table 1 gives the derivative $\psi_{\theta \prime \prime \prime}$ of the vertices and the lowest SIF $K_{1}$ along the line 3 corresponding to the different thickness.

\begin{tabular}{|c|c|c|c|c|c|}
\hline$(h / a)$ & & 3 & 4 & 5 & Ref. $\{12]\left(K_{l}\right)$ \\
\hline \multirow{3}{*}{0.5} & $\psi_{\forall \rightarrow \eta n}$ & $0.400878 \mathrm{e}-4$ & $0.374249 \mathrm{e}-4$ & $0.369290 \mathrm{e}-4$ & \multirow{3}{*}{34.18575} \\
\hline & $\alpha_{1}$ & 59.389364 & 55.444255 & .54 .709682 & \\
\hline & $K_{1}$ & 37.216765 & 34.744366 & 34.284209 & \\
\hline \multirow{3}{*}{1.0} & $\psi_{H-\eta \eta}$ & $0.501775 \mathrm{e}-5$ & $0.512247 \mathrm{e}-5$ & $0.513005 e-5$ & \multirow{3}{*}{9.56300} \\
\hline & $\alpha_{1}$ & 7.433703 & 7.588846 & 7.600080 & \\
\hline & $\Lambda_{1}$ & 9.316766 & 9.511198 & 9.525287 & \\
\hline
\end{tabular}

Example 2 A pressurized cylindrical shell with a longitudinal crack

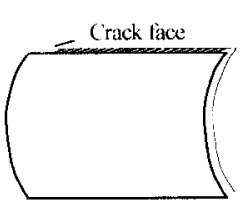

(a) A quarter domain

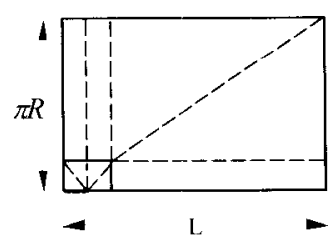

(b) FEMOL meshes

(b) Fig. $4 \mathrm{~A}$ finite length problem

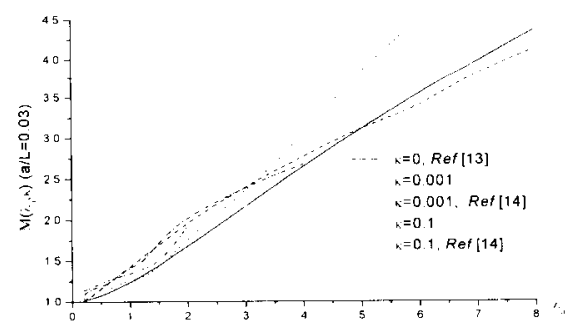

Fig. $5 M\left(\lambda_{a}, \kappa\right)(a / L=0.03)$

In this example, as Fig. 3 shows, we consider a pressurized cylindrical shell with a longitudinal crack. In most references, the SIF $K_{,}{ }^{\mid 14]}$ and the bulging factor $M$ are defined as

$$
K_{l}=K_{l}^{h}+K_{l}^{*}=\left(K_{m m}+K_{b m}\right) \frac{q_{0} R}{h} \sqrt{\pi a}, \quad M=K_{m m}+K_{b m}
$$

the necessary constants are $\lambda_{a}=\sqrt[4]{12\left(1-v^{2}\right)} a / \sqrt{R h}, \kappa=D / C a^{2}, E=2 \times 10^{\circ}, v=0.3, a=1, p=2$.

Consider a finite problem as $a / L=0.03$, a quarter body is taken in computing by using the symmetry as Fig. 4(a) shows, the FEMOL meshes as Fig. 4(b) shows. Table 2 gives the computed results of bulging factor and the compare with Ref. [13] and Ref. [14]. Fig. 5 gives the compared curves according to the Table 2 . 
Table 2 Computed results of the bulging factor $(a / L=0.03)$

\begin{tabular}{|c|c|c|c|c|c|}
\hline \multirow{2}{*}{$\lambda_{a}$} & \multirow{2}{*}{$\frac{0}{\text { Ref. [13] }}$} & \multicolumn{2}{|c|}{0.001} & \multicolumn{2}{|c|}{0.1} \\
\hline & & $M\left(\lambda_{a}, \kappa\right)$ & Ref. $[14]$ & $M\left(\lambda_{a}, \kappa\right)$ & Rel: [14] \\
\hline 0.2 & 1.0137 & $1.012 \overline{392}$ & 1.04 & 1.088197 & 1.13 \\
\hline 0.4 & 1.0483 & 1.096604 & 1.09 & 1.177397 & 1.19 \\
\hline 0.6 & 1.0985 & 1.214868 & 1.14 & 1.192948 & 1.26 \\
\hline 1.0 & 1.2329 & 1.426322 & 1.27 & 1.434653 & 1.34 \\
\hline 1.2 & 1.3122 & 1.533991 & 1.28 & 1.453101 & 1.39 \\
\hline 1.6 & 1.4866 & 1.739218 & 1.47 & 1.811987 & 1.52 \\
\hline 2.0 & 1.6743 & 1.974668 & 1.78 & 2.043780 & 1.93 \\
\hline 3.0 & 2.1636 & 2.406444 & 2.34 & 2.400734 & \\
\hline 4.0 & 2.6469 & 2.766640 & 3.01 & 2.674663 & \\
\hline 5.0 & 3.1096 & 3.127763 & 3.86 & & \\
\hline 6.0 & 3.5459 & 3.366533 & 4.49 & & \\
\hline 7.0 & 3.9685 & 3.829041 & & & \\
\hline 8.0 & 4.3667 & 4.121953 & & & \\
\hline
\end{tabular}

\section{CONCLUDING REMARKS}

To sum up and end up this paper, the following conclusions can be drawn:

(1) Generality: The present algorithm is a general approach and is applicable to any two dimensional singular problems, if only the lowest eigen-value $\lambda_{1}$ is given.

(2) Accuracy: Ref. [4] gives some rules and tips to the ODE solving (COLSYS) that one is 'Do not impose tolerances on derivatives for singular solutions'. These rules have been broken down by the singular mapping technique. The tolerances can be confined on the derivatives and the accuracy is fully controlled by the user with a desired error tolerance specified to the solver. This guarantees that the solutions of singular problem are in the desired accuracy.

(3) Efficiency: By using the technique, FEMOL elements can be constructed with the desired singular behavior built-in. The computation is speeded up and the accuracy is improved.

(4) Strongpoint: In FEMOL, the present singular line mapping is not limited to the crack case. It is applied to notches with any opening angles. The accurate derivatives around the vertices can be computed.

(5) Lowest SIF: SIF can be easily solved from the accurate derivatives along the different lines.

\section{Acknowledgments}

This work was supported by the National Natural Science Foundation of China.

\section{REFERENCES}

1. Si Yuan, A Proceedings of 1st National Conference on Analytical and Numerical Combined Methods, Hunan (1990) p. 132-136 (in Chinese).

2. Si Yuan and Jianling Gao, Proceedings of International Conference on EPMESC, Macau, 3 (1990) p. 517 526.

3. Si Yuan, Chinese Journal of Numerical Mathematics and Applications, 15, 1 (1993) p. 45-59.

4. Si Yuan, The Finite Element Method of Lines: Theory and Applications, Science Press, Beijing,-New York (1993).

5. U. Ascher, et al, ACM Tranaction of Mathematical Software, 7, 2 (1981) p. 209-222.

6. U. Ascher, et al, ACM Tranaction of Mathematical Software, 7, 2 (1981) p. 223-229.

7. Si Yuan. Computational Structural Mechanics and Applications, 10, 1 (1993) p. 118-122 (in Chinese).

8. Si Yuan, Science in China, Series A. 23, 5 (1993) p. 552-560 (in Chinese).

9. Yongjun Xu and Si Yuan, International Journal of Fracture, Vol. 18, (1996) p. 373-381.

10. Yuan Si, Computational Mechanics '92, Proc. Of ICCES, Hong Kong, (1992) p. 47.

11. Xu Yongjun and Yuan Si, Engineering Mechanics(Spe. Iss), (1999) p. 85-91 (in Chinese)

12. Yingzhi Li and Chuntu Liu, ACTA MECHANICA SINICA, 4 (1983) p. 366-375 (in Chinese)

13. F. Erdogan and J. J. Kibler, Int. J. of Fracture Mechanics, 5, 3, (1969) p. 229-237.

14. Chuntu Liu and Xijia Wu, ACTA MECHANICA SINICA, 19, 4 (1987) p. 366-375 (in Chinese). 
Fracture and Strength of Solids IV

10.4028/www.scientific.net/KEM.183-187

Singularity Intensity Factor Calculation in Plates and Shells Based on the Singular Line Mapping Technique in FEMOL

10.4028/www.scientific.net/KEM.183-187.151

\section{DOI References}

[5] U. Ascher, et al. ACM Tranaction of Mathematical Sofiware, 7, 2 (1981) p. 209-222. doi:10.1145/355945.355950

[13] F. Erdogan and J. J. Kibler, mt. J. of Fracture Mechanics, 5,3, (1969) p.229-237. doi:10.1007/BF00184614 